\title{
SARAH 4: A tool for (not only SUSY) model builders
}

\author{
Florian Staub \\ Bethe Center for Theoretical Physics $\&$ Physikalisches Institut der Universität Bonn, \\ Nußallee 12, 53115 Bonn, Germany
}

\begin{abstract}
We present the new version of the Mathematica package SARAH which provides the same features for a non-supersymmetric model as previous versions for supersymmetric models. This includes an easy and straightforward definition of the model, the calculation of all vertices, mass matrices, tadpole equations, and self-energies. Also the two-loop renormalization group equations for a general gauge theory are now included and have been validated with the independent Python code PyR@TE. Model files for FeynArts, CalcHep/CompHep, WHIZARD and in the UFO format can be written, and source code for SPheno for the calculation of the mass spectrum, a set of precision observables, and the decay widths and branching ratios of all states can be generated. Furthermore, the new version includes routines to output model files for Vevacious for both, supersymmetric and non-supersymmetric, models. Global symmetries are also supported with this version and by linking Susyno the handling of Lie groups has been improved and extended.
\end{abstract}

\section{Program Summary}

Manuscript Title: SARAH 4: A tool for (not only SUSY) model builders

Author: Florian Staub

Program title: SARAH

Programming language: Mathematica

Computers for which the program has been designed: All Mathematica is available for

Operating systems: All Mathematica is available for

Keywords: Model building, gauge theory, Renormalization group equations, Vacuum constraints

CPC Library Classification: 11.1, 11.6

Reasons for the new version: New features in the definition of models and a full support of nonsupersymmetric models. New output for Vevacious.

Solution method: Non-supersymmetric models are supported by the new possibility to define not only chiral superfields but also component fields. The renormalization group equations (RGEs) for a nonsupersymmetric models are calculated by using the generic formulae for a general quantum field theory.

Unusual features: Calculation of non-supersymmetric RGEs includes effects of kinetic mixing as well as gauge dependence of running vacuum expectation values. SARAH is the first tool which can automatically create model files for Vevacious. Fully automatized derivation of all terms in the Lagrangian which are fixed by gauge invariance.

Restrictions: Only renormalizable terms in the Lagrangian are supported. No support of fields with spin 2 or $3 / 2$.

Summary of revisions: support of non-supersymmetric models; calculation of renormalization group equations for a general gauge theory; link to Susyno for handling of non-SU(N) gauge groups; support of global symmetries; output of model files for Vevacious; support of aligned VEVs; calculation of gauge dependent parts of RGEs for VEVs in running of supersymmetric and non-supersymmetric models.

Does the new version supersede the previous version?: Yes, the new version includes all known 
features of previous versions but provides also the new features mentioned above.

Running time: Loading the Standard Model: 1.6s; calculation of all vertices: 11.8s; calculation of all RGEs: $130.2 \mathrm{~s}$; output for Vevacious model files: 0.1s; output of model files in UFO format: 0.8s; output of model files for FeynArts: 0.1s; output of model files for CalcHep: 0.8s; output of model files for WHIZARD: 3.5s; writing of source code for SPheno: 34.5s. All times measured on Lenovo X220 with Intel(R) Core(TM) i7-2620M CPU @ 2.70GHz.

\section{Introduction}

Previous versions of SARAH 1-4 ] have been optimized for an easy, fast and exhaustive study of supersymmetric (SUSY) models beyond the minimal supersymmetric standard model (MSSM). The range of models covered by SARAH is very broad and it has been successfully used to study many different SUSY models: singlet extensions with and without $\mathrm{CP}$ violation [5 7], models with $R$-parity violation [8, 9], different kinds of seesaw mechanisms [10 12], models with extended gauge sectors at intermediate scales [13, 14] or the SUSY scale [15, 16], models with Dirac Gauginos [17, 18], and even more exotic ones [19].

The emphasis in the new version has been to push also non-supersymmetric (non-SUSY) models beyond the standard model (BSM) to the same level of automatization. For this purpose, the input format has been adjusted to support the definition of fermionic and scalar fields separately beside the definition of superfields only used so far. The calculation of vertices, mass matrices, tadpole equations and one-loop corrections to 1and 2-points functions have always been performed in component fields and the corresponding routines can be used now for SUSY as well as non-SUSY models. In contrast, the generic formulae for the calculation of SUSY RGEs are not valid in the non-SUSY case. Therefore, new routines for the calculation of the two-loop $\beta$-functions in a general quantum field theory have been implemented.

For SUSY and non-SUSY models the calculated vertices can be used to write model files for CalcHep/ CompHep [20, 21], FeynArts/FormCalc [22, 23], WHIZARD/O'Mega [24, 25] as well as in the UFO format [26] which can be handled for instance by MadGraph 5 27], GoSam 28] and Herwig++ 29 31]. In addition, due to the output of Fortran source code for SPheno [32, 33] the calculation of the one-loop corrected mass spectrum, branching ratios and decays widths of all states is possible. Also precision observables are calculated and an easy link to HiggsBounds [34, 35] and HiggsSignals [36] exists.

Furthermore, global symmetries are supported in the new version and a link between SARAH and Susyno [37] has been established. By using routines of Susyno for the handling of Lie groups, SARAH is no longer restricted to $U(1)$ and $S U(N)$ gauge groups and the non-fundamental irreducible representations (irreps) of unbroken gauge groups can be treated in a more convenient way since it no longer necessary to express them as tensor products of the fundamental representation.

Finally, SARAH 4 can also produce model files for Vevacious [38]. The combination SARAH-SPhenoVevacious provides the possibility to check the one-loop effective potential of a given model and parameter point for the global minimum. In those checks the possibility of dangerous vacuum expectation values (VEVs) of charged and/or colored scalars can be taken into account and the life-time of meta-stable vacua can be calculated using Cosmotransitions 39].

This paper is organized as follows: the basics about the download and installation are shown in sec. 2 and in sec. 3 we present the changes in the input format of SARAH which were necessary to support global symmetries, to link Susyno and to handle non-SUSY fields in a convenient way. In sec. 4 we discuss the calculation of RGEs for a general quantum field theory. In sec. 5 we explain the different possibilities to calculate the mass spectrum and other observables with SPheno and how to pass this information to Monte Carlo (MC) tools. In sec. 6 we introduce the interface to Vevacious before we conclude in sec. 7. Other new features in SARAH 4 as well as an overview about the most important commands and existing models are given in the appendix.

\section{Download and Installation}

SARAH 4 has been successfully tested with Mathematica versions 7 to 9, but the support of earlier versions of Mathematica has stopped. It can be downloaded from 
http://sarah.hepforg.org

After downloading the tar-file, the package is extracted via

tar -xf SARAH-4.0.0.tar.gz

We are going to assume in the following that SARAH has been installed in the directory [\$SARAH-Directory] .

To load SARAH, start Mathematica and execute

$<$ [\$SARAH-Directory] / SARAH.m

Afterwards, a model (called [\$MODEL] in the following) which is already included in SARAH is initialized via Start $["[$ MODEL]"];

For instance, to load the MSSM [\$MODEL]=MSSM is used. For an overview of commands which can be used to study the model after the initialization we refer to Appendix B and to the manual for more details. A list of all models delivered with SARAH 4 is given in Appendix C and the implementation of new models was discussed in Refs. 2, 3]. We concentrate in the following on new features in SARAH 4.

\section{New Possibilities in the Definition of a Model}

\subsection{Global Symmetries}

Beginning with SARAH $4 Z_{N}$ and $U(1)$ global symmetries can be defined. For this purpose, a new array Global has been introduced:

Global $[[1]]=\{Z[2]$, RParity $\}$

Global $[[2]=\{\mathrm{U}[1]$, PecceiQuinn $\}$

First, the kind of the symmetry is defined and afterwards a name is given to the symmetry. In principle, up to 99 different global symmetries can be added for one model. The charges of all fields with respect to these groups are included in the definition of each field as discussed in sec. 3.3. By convention, all $Z_{N}$ symmetries are always taken to be multiplicative symmetries. This means, the charges $Q$ of additive symmetries have to be given as $\exp (2 i \pi Q)$. In addition, there is one special keyword to name a $U(1)$ : if the symmetry is called RSymmetry also the $R$ charges of the SUSY coordinates are included in the checks for charge conservation. See for example the minimal $R$-symmetric SSM (MRSSM) which is included in SARAH.

The global symmetries are taken into account during the following operations:

1. Checking the given (super)potential for charge conservation: all terms in the potential and superpotential defined by the user are checked since SARAH 2 for gauge invariance. In the new version also the global symmetries are considered and if terms violate any of these symmetries a warning is printed.

2. Checking for all possible terms in the (super)potential: starting with version 3 SARAH provides routines which check for all (renormalizable) terms in the superpotential for the given superfield content which are in agreement with gauge invariance and $R$-parity. Now, all global symmetries are taken into account during these checks. Also similar routines exist which check for all possible (renormalizable) terms in the potential of a non-SUSY model. To perform these checks run CheckModel after the initialization of a model.

3. Adding SUSY soft-breaking masses for scalars: SARAH adds automatically for a SUSY model the soft mass terms for all scalars. These are usually of the form $m^{2}\left|\phi_{i}\right|^{2}$ where $i$ labels the different scalars. In the case that fields have the same quantum number, also terms of the form $m^{2} \phi_{i} \phi_{j}^{*}+h . c$. can exist in principle. So far, SARAH checked only the gauge quantum numbers and $R$-parity to include/exclude such terms. Now, all global symmetries are considered. 
4. Output for MicrOmegas: the model files for CalcHep which are supposed to be used with MicrOmegas must contain the information about a conserved $Z_{2}$ symmetry. By default, this symmetry is now taken to be the one called RParity in Global. If no $Z_{2}$ symmetry with this name is exists, the first $Z_{2}$ symmetry defined in Global is used instead.

So far $R$-parity has been the only discrete symmetry which was supported by SARAH. For this purpose, the $R$-parity of each particle has been given in the particle definitions in the file particles.m. This entry has been become obsolete and is ignored now. In the same way, the flag RParityConservation $=$ True/False which could be added to the model file has no longer any effect, but it is checked if a global symmetry called RParity is present or not.

\subsection{Gauge Sector}

The handling of Lie groups has been significantly improved by linking routines from the Mathematica package Susyno to get the representation matrices, Clebsch-Gordan coefficients (CGCs) and gauge group constants. With doing this, not only $U(1)$ and $S U(N)$ groups can be handled, but also $S O(N), S p(2 N)$ and expectational groups are supported. Non- $S U(N)$ gauge groups are defined in the same way as $S U(N)$ ones:

Gauge $[[1]]=\{\mathrm{X}, \mathrm{U}[1]$, extra, $\mathrm{g} 1, \mathrm{False}\}$;

Gauge $[[2]]=\{\mathrm{G} 10, \mathrm{SO}[10], \mathrm{SOGUT}, \mathrm{g} 2, \mathrm{False}\}$;

First, the names of the gauge fields are given, then the kind of the group is defined. The third and fourth entry define the name of the gauge group and of the corresponding gauge coupling. In the last entry it is defined if the sums over charge indices of the gauge group should be expanded (True) or not (False). The last entry has no effect for Abelian groups.

The names of the gauge fields are derived as follows from the first entry of each gauge group: gauginos start with $f$ (i.e. $f X, f G 10$ ), the names of vector bosons are extended by $V$ as first letter (i.e. VX, VG10) and the corresponding ghosts start with a $\mathrm{g}$ (i.e. gX, gG10). In the case that the gauge groups carry a charge under a global symmetry, these are added at the end. Here, the same conventions are used as for matter fields discussed in the next section.

\subsection{Matter Fields}

\subsubsection{Superfields, Scalars and Fermions}

SARAH has so far only supported chiral superfields in the matter sector which were defined in an array called Fields. This has been a limitation for the study of non-SUSY fields in contrast to other tools like LanHEP [40-43] or FeynRules [44, 45] which support the definition of component fields. This restriction is no longer present, but three possibilities to define matter fields exist now: SuperFields, FermionFields and ScalarFields. In this way it is now as simple to define a non-SUSY model in SARAH as it has been for SUSY models all the time. For backward compatibility, Fields is still supported by SARAH but all model files delivered with SARAH have been changed to the new format. We have to stress that the conventions of SuperFields are slightly different to those of Fields: the first and third entry have been exchanged. In addition, the transformation properties with respect to the global symmetries are added after the quantum numbers with respect to the gauge groups.

Conventions to define global charges. If only one quantum number is given per superfield per global symmetry this number is used for the superfield itself but also for the scalar and fermionic component. To define a symmetry which distinguishes between the superfield and its components, a list with three entries has to be given. For chiral superfields, the first entry is the charge for the superfield, the second for the scalar component, the third for the fermionic component. For vector superfield, the second entry refers to the gaugino, the third to the gauge boson. A convenient possibility might be to define new variables for such entries, e.g. 


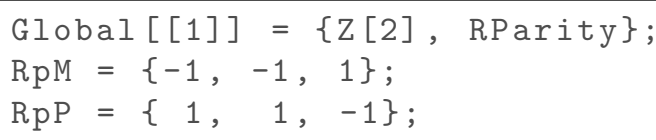

with RpM for $R$-parity - 1 and RpP for $R$-parity +1 .

The charges under global symmetries defined for the gauge eigenstates are added during all rotations also to the mixed eigenstates as long as all fields in the basis of the rotation have the same charge. If the charges are different, the rotated fields don't get any charge under that global symmetry but a warning is printed. For example, if someone would have chosen the standard definition of $R$-parity but mixes sneutrinos and Higgs fields, the corresponding mass eigenstate wouldn't have a definite $R$-parity.

Definition of fields. For a realistic model we define first the SM gauge groups using the conventions of sec. 3.2 via

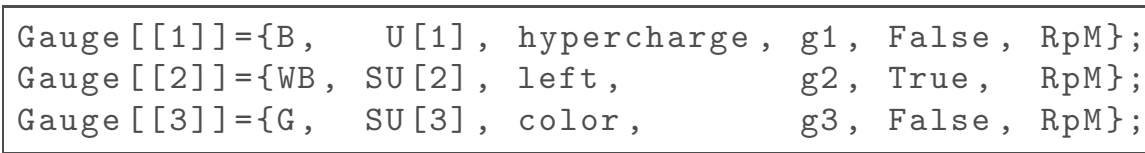

Here, the last entries in each line define the $R$-parity of the corresponding vector superfields and their components. The definition of the quark and Higgs doublets in the MSSM with respect to these gauge groups reads with the new conventions of SARAH 4

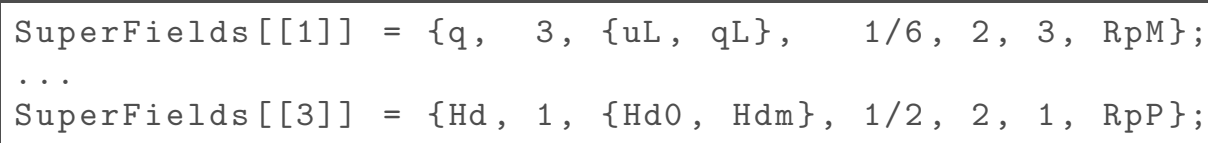

First, a name for the superfield is given, in the second entry the number of generations is set and in the third entry the names for the isospin components are defined. Afterwards, the hypercharge, and the transformation under $S U(2)_{L} \times S U(3)_{C}$ are given, the last entry states the $R$-parity using the variables RpM and RpP defined above. The syntax for scalar and fermion fields in the case of non-SUSY models looks exactly the same:

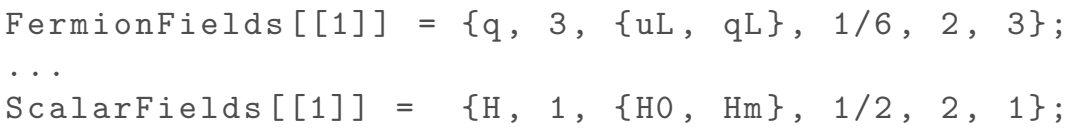

Here, we have not assumed any global symmetry. Thus, the definition of the gauge groups is the same as for the MSSM above just without the last entry in each line. By default all scalars are taken to be complex. To define them as real the name of the field has to be added to the list RealScalars. For instance, a real gauge singlet is added to the model by

ScalarFields $[[2]]=\{\mathrm{S}, 1, \mathrm{~s}, 0,1,1\}$;

Realscalars $=\{\mathrm{S}\}$;

Note, the fermionic components of superfields start with $\mathrm{F}$ and the scalars with S, i.e. the quark doublets stemming from the superfield definition above are called FuL, FdL and the squarks are called SuL, SdL. For fermion and scalar fields defined separately, no renaming takes place but the fields are used exactly as they are defined. It is not possible to use SuperFields and FermionFields or ScalarFields at the same time. If the user wants to define scalars or fermions, all superfields have to be written as components.

\subsubsection{Handling of non-fundamental Representations}

The link to Susyno has opened a new possibility to handle non-fundamental representations of nonAbelian gauge groups: so far these fields have been always treated as tensor products. While this is still 
done for $S U(N)$ gauge groups which get broken, irreps of unbroken gauge groups are written as vectors with the appropriate dimension. This has especially advantages for models which contain color sextets: for these models it is possible now to write model files in the UFO format which can be used with MadGraph 5. The same would hold for all other color representations like decuplets, but those are not yet supported by any $\mathrm{MC}$ tool.

To treat non-fundamental irreps as vectors under the corresponding gauge group, a major rewriting of core routines of SARAH has been necessary. This has several consequences:

- The list of all existing particles does not only contain the indices of each particle, but also the dimension of each index is explicitly given. For instance, the entry of the d-squarks in Particles [EWSB] changed from

$\{\mathrm{Sd}, 1,6, \mathrm{~S},\{$ generation, $\operatorname{color}\}\}$

to

$\{\mathrm{Sd}, 1,6, \mathrm{~S},\{\{$ generation, 6$\},\{\operatorname{color}, 3\}\}$

- There is no longer a special name for charge indices of the adjoint representation (so far a $<>$ index), i.e. the entry of the gluon in Particles [EWSB] has changed from

$\{\mathrm{VG}, 1,1, \mathrm{~V},\{$ acolor, lorentz $\}$

to

$\{\mathrm{VG}, 1,1, \mathrm{~V},\{\{\operatorname{color}, 8\},\{$ lorentz, 4\}\} $\}$

- There is just one charge index per particle per unbroken gauge group, i.e. non-fundamental irreps carry less indices than before. For instance, a fermionic color sextet which was so far treated as $3 \times 3$ matrix with two color indices of dimension 3

$\{\mathrm{Fsix}, 1,1, \mathrm{~F},\{\operatorname{col} 0 \mathrm{r}, \operatorname{colorb}\}$

is now defined internally as

$\{$ Fsix $, 1,1, F,\{\{\operatorname{col} o r, 6\}\}\}$

\subsubsection{Defining Matter Interactions}

The syntax to define the superpotential has been simplified. It is no longer necessary to give it as a list of terms but a more intuitive form as sum is possible. For instance, instead of the former definition of the superpotential of the MSSM

SuperPotential $=\{\{\{1, Y u\},\{u, q, H u\}\},\{\{-1, Y d\},\{d, q, H d\}\}$,

$\{\{-1, \mathrm{Ye}\},\{\mathrm{e}, 1, \mathrm{Hd}\}\},\{\{1, \backslash[\mathrm{Mu}]\},\{\mathrm{Hu}, \mathrm{Hd}\}\}\}$;

the short form

SuperPotential = Yu u.q.Hu $-Y d d . q . H d-Y e$ e.l.Hd $+\backslash[\mathrm{Mu}] \mathrm{Hu} \cdot \mathrm{Hd}$;

can be used.

In the same way, the potential for non-SUSY models is defined as sum of terms 


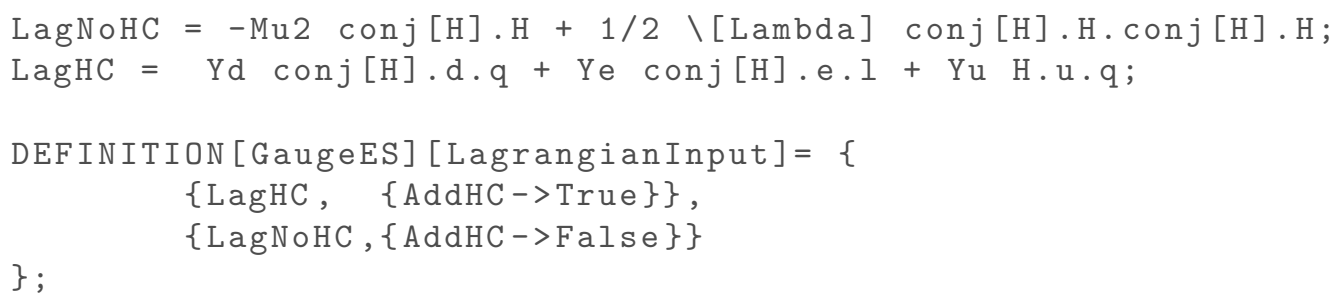

Here, we defined all interactions in terms of gauge eigenstates (GaugeES) and all rotations to the mass eigenstates are performed by SARAH. In addition, there exists the option that SARAH adds automatically the Hermitian conjugated for the given part of the Lagrangian (AddHC->True).

It is neither necessary for the superpotential nor for terms added via DEFINITION [_] [LagrangianInput] to define any index structure. This task is automatically performed by SARAH: it adds generation indices if necessary to all fields and parameters and contracts all charges using either the Kronecker delta (Delta), the antisymmetric tensor (epsTensor) or CGCs. CGCs are a new feature in SARAH 4 since in previous versions it has been sufficient just to use the Kronecker delta and antisymmetric tensor because of the treatment of all irreps as tensor product. The CGCs which are now used for interactions involving higher dimensional irreps are parametrized as follows:

CG [group, dnykin] [indices]

First, the gauge group is given, afterwards the Dynkin labels of all involved irreps are stated and finally the indices are listed. For instance, the interaction between a scalar color sextet (six) and two fermionic triplets which appear in several generations $(t)$ is defined in the input by the user just as

Ys $\operatorname{six} \cdot \mathrm{t} \cdot \mathrm{t}$

and internally expanded by SARAH to

Ys $[\operatorname{gen} 2, \operatorname{gen} 3] * \mathrm{CG}[\mathrm{SU}[3],\{\{0,2\},\{1,0\},\{1,0\}\}][\operatorname{col} 1, \operatorname{col} 2, \operatorname{co} 3] *$

$\operatorname{six}[\{\operatorname{col} 1\}] * t[\{\operatorname{gen} 2, \operatorname{col} 2\}] * t[\{\operatorname{gen} 3, \operatorname{col} 3\}]$

The numerical values for all CGCs are calculated by Susyno. The user can check the array

SA'LagrangianContractions

to see what contractions have been used by SARAH for each term.

SARAH takes by default the first gauge invariant contraction of indices it finds. That's usually the simplest contractions which exists. That means that always co- and contravariant indices are contracted which are next to each other, e.g. $\phi^{i j} \Psi_{k} \Psi_{l} \delta_{j k} \delta_{i l}$ is used but not $\phi^{i j} \Psi_{k} \Psi_{l} \delta_{i k} \delta_{j l}$. Furthermore, it is also assumed that the contraction is not a sum of different products involving Kroneckers deltas and antisymmetric tensors but just one term. For instance, $\phi^{i} \phi_{j} \phi_{k} \phi^{l}$ is contracted with $\delta_{i j} \delta_{k l}$ while another gauge invariant form would be the result of the completeness relation of $S U(N)$ gauge groups: $2 \delta_{i l} \delta_{j k}-\frac{2}{N} \delta_{i l} \delta_{j k}$. Since several possibilities to contract the indices of one term might exist, it is also possible that the user can define a contraction for each term which is then used by SARAH.

Example. It might for instance be necessary for (renormalizable) interactions which are an effect of fields integrated out to define the contractions by hand. For instance, D-terms of heavy vector superfields can give rise to $\left|H_{u}^{\dagger} \sigma_{a} H_{u}+H_{d}^{\dagger} \sigma_{a} H_{d}\right|^{2}$ [46]. This can be written by making use of the completeness relation of the Pauli matrices as

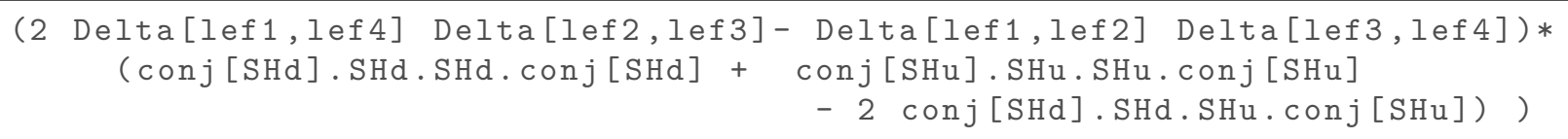


Symmetry of parameters. It is also new that parameters which involve several times the same fields which come in more than one generation are tested for their symmetry: SARAH checks if such a parameter is symmetric, anti-symmetric, hermitian, or anti-hermitian and simplifies expression by using these properties during the different calculations. For instance, Ys introduced above would be defined as symmetric while it would be anti-symmetric if the color triplets would also be doublets under $S U(2)$. The entry Symmetry in parameters.m which has been used in earlier versions to manually define these symmetries is no longer necessary and ignored now.

\subsection{The Lagrangian derived by SARAH}

SARAH derives from the definition of the gauge and matter sector and the (super)potential the full Lagrangian of the model. For this purpose it generates automatically all interactions which are fixed by gauge and, if considered, SUSY invariance, i.e. kinetic terms, self-interactions of vector bosons, D-terms and gaugino interactions. From the kinetic terms also the gauge fixing terms in $R_{\xi}$ gauge are derived and the ghost interactions are calculated. In the case of SUSY models the standard soft terms are added by default, too. Because of this level of automatization the input files are usually very short, they can easily be extended and the possibilities for the user to make errors are reduced. The main restriction to define interactions at the moment is that only renormalizable terms are fully supported. For SUSY models it is possible to define superpotential terms with four superfields. However, only the dimension 5 operators (which enter, for instance, the fermion masses) are derived from that. For the potential itself it is only possible to add renormalizable terms for now.

Examples for the full model files of a SUSY and non-SUSY model in the new format are given in Appendix D (NMSSM) and Appendix E (Standard Model).

\section{Renormalization Group Equations for a general Quantum Field Theory}

\subsection{Non-SUSY RGEs with SARAH}

Since version 2 SARAH calculates the full two-loop RGEs for a SUSY theory based on the generic results given in Ref. [47]. These calculations have been later generalized to the case of several Abelian gauge groups with full kinetic mixing by using the substitution rules presented in Ref. [48]. In version 3.2 the two-loop RGEs for Dirac gauginos based on Ref. [49] have been included. All of these calculations take into account the most general $\mathrm{CP}$ and flavor structure. The gauge dependence of running vacuum expectation values (VEVs) is also included, see Appendix A.4.

SARAH 4 calculates the RGEs for a general quantum field theory with the same precision as it is done for SUSY models based on the generic results of Refs. 50 53. Also the impact of kinetic mixing for non-SUSY models has been included by using the corresponding rules which became recently available in Ref. 54. Finally, the gauge dependence in the running of the VEVs is now included by using the results of Ref. [55].

The calculation of the RGEs can be started after the initialization of a model via CalcRGEs [Options];

This is the same command which is also used to get the SUSY RGEs, i.e. SARAH checks which calculation is valid for the given model. The different options are

- TwoLoop, Value: True or False, Default: True

Defines, if also the two-loop RGEs should be calculated.

- ReadLists, Value: True or False, Default: False

Defines, if previously calculated RGEs should be loaded. 
- VariableGenerations, Value: List of particles, Default: \{\}

Defines, that the generations of specific fields should be treated as variable. For this purpose variables $\mathrm{nG}[\mathrm{X}]$ are introduced, where $\mathrm{X}$ is the name of the superfield (SUSY model) or fermion/scalar (nonSUSY model).

- NoMatrixMultiplication, Values: True or False, Default: False

This can be used to switch on/off matrix multiplication in the simplification of the RGEs.

- IgnoreAt2Loop, Values: a list of parameters, Default: \{\}

Can be used to define parameters which should be neglected during the calculation of the two-loop $\beta$-functions.

- WriteFunctionsToRun, True or False, Default: True Defines, if a file should be written to evaluate the RGEs numerically in Mathematica, see sec. 4.3

The results are saved in three dimensional arrays: the first entry is the name of the considered parameter, the second entry is the one-loop $\beta$-function and the third one is the two-loop $\beta$-function. For non-SUSY models the different generic structures are saved in

- Gij: Anomalous dimensions of all fermions and scalars

- BetaGauge: Beta functions of all gauge couplings

- BetaLijkl: Beta functions of all quartic scalar couplings

- BetaYijk: Beta functions of all interactions between two fermions and one scalar

- BetaTijk: Beta functions of all cubic scalar interactions

- BetaMuij: Beta functions of all bilinear fermion terms

- BetaBij: Beta functions of all bilinear scalar terms

- BetaVEV: Beta functions of all VEVs

Examples. Some entries for the Standard Model (SM) look as follows:

- Hypercharge coupling (BetaGauge [[1] $)$

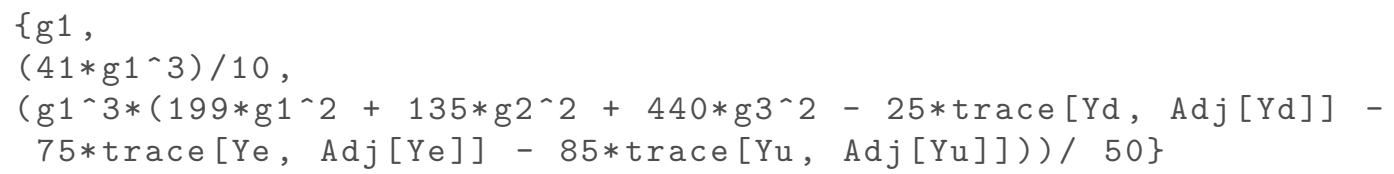

- $\lambda$ coupling (BetaLijkl[[1]]; only the one-loop part is shown)

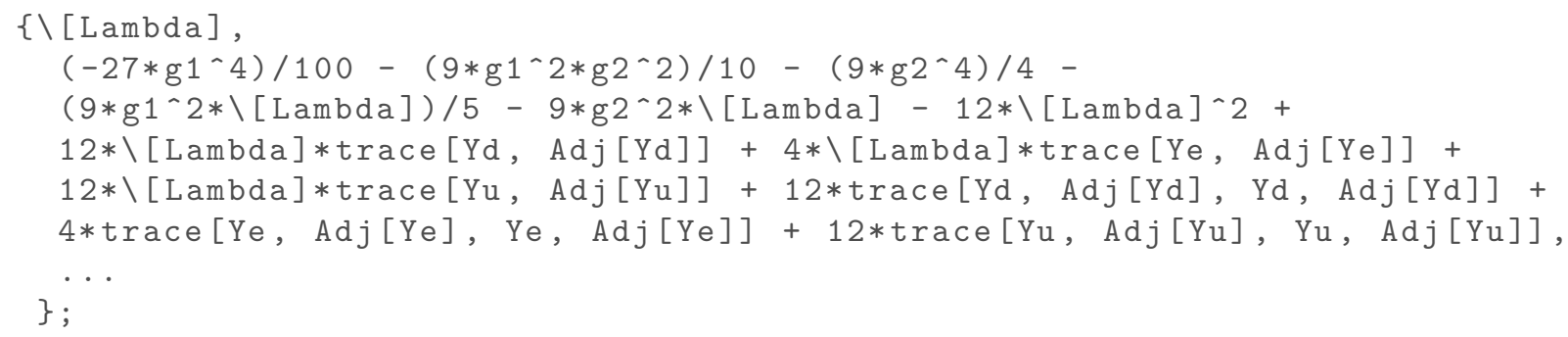

- Electron Yukawa coupling (BetaYijk[[1]]; only the one-loop part is shown) 


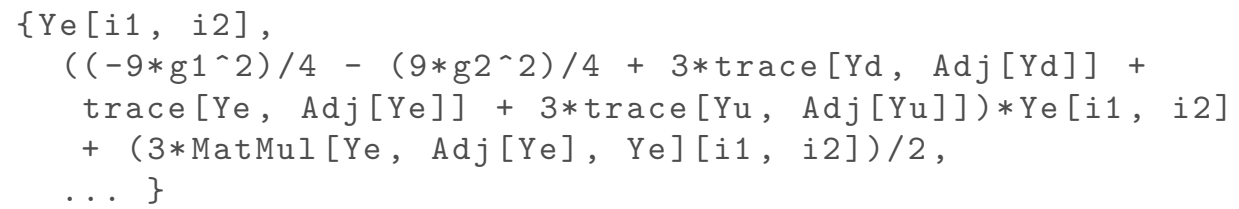

- If the user wants to see the impact on the number of generations of quarks on the running gauge couplings, it is possible to run CalcRGEs [VariableGenerations->\{d, q, $\mathrm{u}\}$ ]. The $\beta$-functions involve then variables $\mathrm{nG}[\mathrm{X}]$ which correspond to the chosen number of generations for those particles, e.g. BetaGauge reads

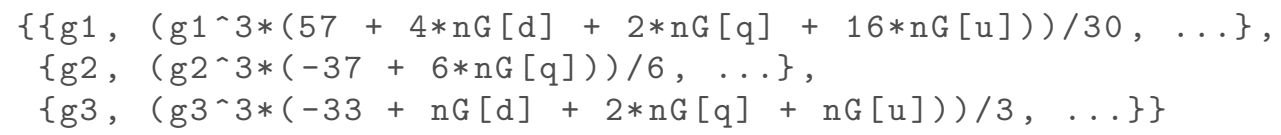

\subsection{Validation}

In literature the full two-loop RGEs for a non-SUSY model beyond the SM are hardly available. Especially, parameters with dimension of mass like cubic scalar interactions are usually not calculated with this precision. This has been one of the reasons to develop a completely independent code in python. The result is the public package PyR@TE [56] which has been used to cross check the results obtained by SARAH. We found full agreement between the results by SARAH and PyR@TE. In addition, the results have been checked against the literature for those models and terms for which a reference was available. We found widely agreement with the literature but could also spot some mistakes in the references. Details about all cross checks are given in Ref. [56].

\subsection{Numerical Evaluation of the RGEs with Mathematica}

SARAH 4 does not only save the calculated RGEs in the internal format in separated text files in [\$SARAH-Directory]/Output/ [\$MODEL]/RGEs, but writes in addition a file which can be used for a numerical evaluation of the SUSY and non-SUSY RGEs in Mathematica. This file is stored in the same directory as the other results for the RGEs and is called RunRGEs.m. It provides all RGEs in a format which is understood by Mathematica and a function called RunRGEs for the numerical evaluation based on NDSolve. The syntax of this function is as follows:

RunRGEs [initialization, $\log ($ start), log(end), Options];

First, a list with the non-zero values of parameters are given. The second and third entry define the logarithm of the scale where the running starts and stops. The option defines if two-loop contributions should be included or not TwoLoop -> True/False. By default the two-loop parts are taken into account. RunRGEs returns the InterpolationFunction obtained by NDSolve.

Example. A one-loop running of the gauge couplings from $1000 \mathrm{GeV}$ to $10^{16} \mathrm{GeV}$ is performed after loading RunRGEs . m by

$<$ " [\\$SARAH-Directory]/Output/[\\$MODEL]/RGEs/RunRGEs.m" ;

solution $=\operatorname{RunRGEs}[\{\mathrm{g} 1->0.45, \mathrm{~g} 2->0.63, \mathrm{~g} 3->1.04\}, 3,16$, TwoLoop->False $]$;

Here, we stored the output of RunRGEs as variable, which can be used as follows

$\{\mathrm{g} 1[16], \mathrm{g} 2[16], \mathrm{g} 3[16]\} /$. solution [[1]];

Plot $[\mathrm{g} 1[\mathrm{x}] /$. solution $[[1]],\{\mathrm{x}, 3,16\}]$;

In the first line, the numerical values of all gauge couplings at $10^{16} \mathrm{GeV}$ are shown, in the second line, the running of $g_{1}$ between $10^{3}$ and $10^{16} \mathrm{GeV}$ is plotted. 


\section{Mass Spectrum Calculation of non-SUSY Models with SPheno and Interface to Monte Carlo Tools}

\subsection{SPheno and non-SUSY Models}

SARAH 3 has been the first 'spectrum-generator-generator': using the derived information about the mass matrices, tadpole equations, vertices, loop corrections and RGEs for the given model SARAH writes Fortran source code for SPheno. Based on this code the user gets a fully functional spectrum generator for the given model. The features of a spectrum generator created in this way are (i) a precise mass spectrum calculation using two-loop RGEs and full one-loop corrections to all masses, (ii) the calculation of branching ratios and decay width of all SUSY and Higgs fields as well as (iii) a prediction for precision observables like $b \rightarrow s \gamma, g-2$ and $B_{s, d}^{0} \rightarrow l_{i} \bar{l}_{j}[57$ ]. In addition, SPheno modules created by SARAH can write input files for HiggsBounds and HiggsSignals.

All of these possibilities are now also available for non-SUSY models as well. For non-SUSY models a few adjustments have happened.

1. SPheno calculates the SM gauge and Yukawa couplings from the experimental data. The corresponding routines written by SARAH assumed so far that two Higgs VEVs $\left(v_{d}, v_{u}\right)$ are presented. In SARAH 4 these routines got adjusted to support also models with just one Higgs VEV like the SM or simple extensions of it.

2. In the case of a non-SUSY model, the translation from $\overline{\mathrm{MS}}$ to $\overline{\mathrm{DR}}$ parameters as well as the calculation of SUSY thresholds is skipped.

3. Finally, all self-energies are calculated in $\overline{\mathrm{MS}}$ instead of $\overline{\mathrm{DR}}$ by using the new flexibility of the loop calculations shown in Appendix A.5.

To create the SPheno output for a given model run in Mathematica

$<<$ SSARAH-Directory] / SARAH . m;

Start [" [\$MODEL]"];

MakeSPheno[];

The source code for SPheno will be stored in [\$SARAH-Directory]/Output/[\$MODEL]/EWSB/SPheno/. To compile this code enter the directory of your SPheno installation (which we are going to call [\$SPheno-Directory]), create a new sub-directory and copy the code into this directory:

cd [\$SPheno-Directory]

mkdir [\$MODEL]

cp [\$SARAH-Directory]/Output/[\$MODEL]/EWSB/SPheno/* [\$MODEL]/

Afterwards, the code is compiled via

make Model $=[$ \$MODEL $]$

and a new executable SPheno [\$MODEL] is created. To run this executable an input file LesHouches . in. [\$MODEL] is needed.

./bin/SPheno[\$MODEL] [\$MODEL]/LesHouches.in. [\$MODEL]

The entire output including all parameters, masses, branching ratios and low-energy observables is saved in SPheno.spc. [\$MODEL].

\subsection{Setting the Properties of the SPheno Version}

To create SPheno versions with SARAH all features of the SPheno code are defined by an additional input file SPheno.m of SARAH. The content of this file for non-SUSY models is very similar to the one of SUSY models which has been discussed in great detail in Ref. [58]. We summarize here the main aspects.

In general, there are two different kinds of SPheno versions the user can create: a 'low scale' version which expects all parameters at the renormalization scale, or a 'high scale' version which includes the possibility to perform a RGE running to some higher scale like the GUT or a threshold scale. We are going to use the 
synonym 'GUT scale' in the following even if this scale must not necessarily be connected to a grand unified theory and can even be much lower than $10^{16} \mathrm{GeV}$. For both cases SPheno.m looks slightly different and we are going to discuss both options.

\subsection{1. 'Low Scale' SPheno Version}

The flag to create a low scale SPheno version is OnlyLowEnergySPheno = True. In addition, the list of input parameters (MINPAR), the boundary conditions at the input scale (BoundaryLowScaleInput), the parameters fixed by the tadpole equations (ParametersToSolveTadpoles) and the particles for which 2and 3-body decays are calculated (ListDecayParticles, ListDecayParticles3B) is needed. Altogether, SPheno.m to get a low scale version of the SM looks like

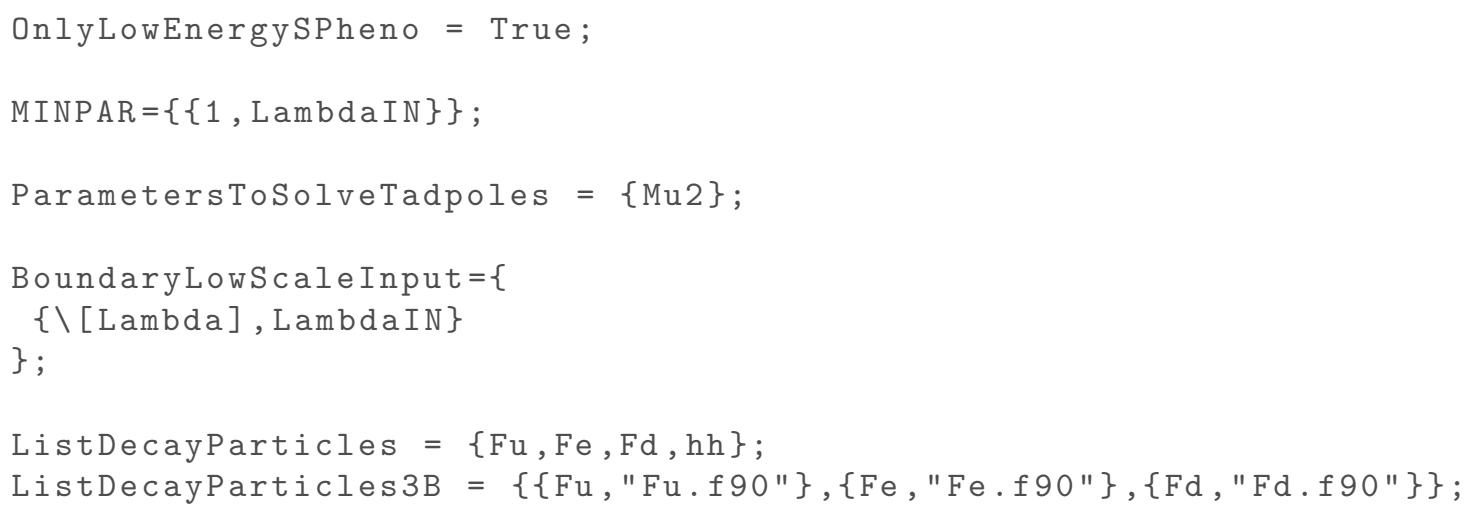

\subsection{2. 'High Scale' SPheno Version}

For a SPheno version which includes the RGE running up to a higher scale, OnlyLowEnergySPheno = False is set. Other differences in comparison to the low scale version is the definition of the renormalization scale (RenormalizationScaleFirstGuess, RenormalizationScale) as well as the boundary conditions at three different scales: (i) at $M_{Z}$ (BoundaryEWSBScale), (ii) at the renormalization scale (BoundaryRenScale), (iii) at the GUT scale (BoundaryHighScale). Here BoundaryRenScale is the equivalent to BoundarySUSYScale for SUSY models. RenormalizationScaleFirstGuess is the renormalization scale used in the first iteration, while RenormalizationScale is used in all following iterations. The main difference is that RenormalizationScale can depend on parameters or masses which are calculated by SPheno while RenormalizationScalefirstGuess must be a constant or completely fixed by the input. In addition, using the variable ConditionGUTscale it is possible to define a condition for the GUT scale, i.e. what relation among parameters should be fulfilled to stop the RGE running and to apply the boundary conditions given in BoundaryHighScale.

With these conventions SPheno.m for the SM including RGE running reads

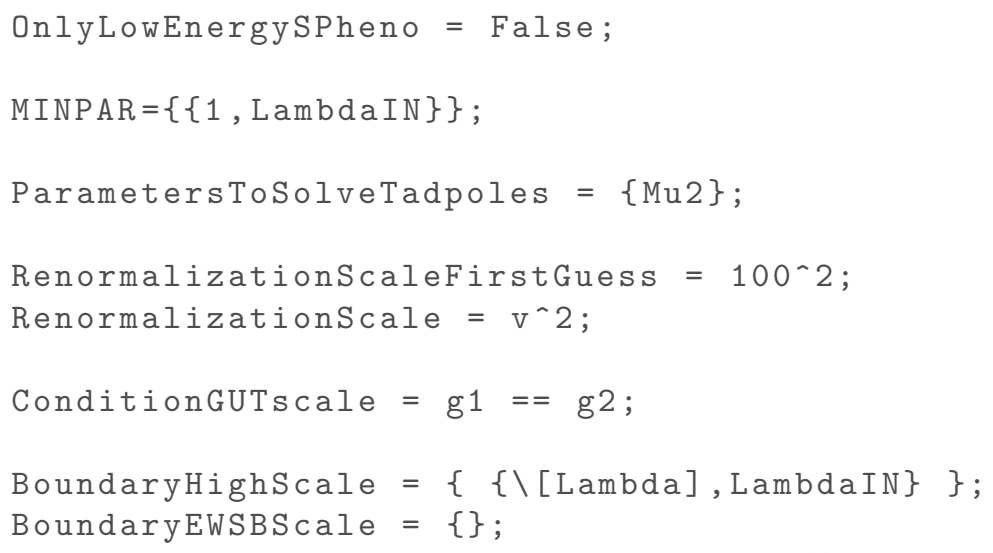


BoundaryRenScale $=\{\}$;

ListDecayParticles $=\{\mathrm{Fu}, \mathrm{Fe}, \mathrm{Fd}, \mathrm{hh}\}$;

ListDecayParticles3B $=\{\{F u, " F u . f 90 "\},\{F e, " F e . f 90 "\},\{F d, " F d . f 90 "\}\}$;

Here we put as condition to stop the RGE running that the running values of $g_{1}$ and $g_{2}$ should be equal. However, in contrast to SUSY models it is not very common to run the RGEs up to the scale where two gauge couplings do meet. Often just the running parameters at some specific scale should be extracted or boundary conditions at some threshold scale where one assumes the theory to break down should be applied. For this purpose, another possibility exists to define the scale where the running stops: it is possible to set the 'GUT scale' to a constant value in the Les Houches input file

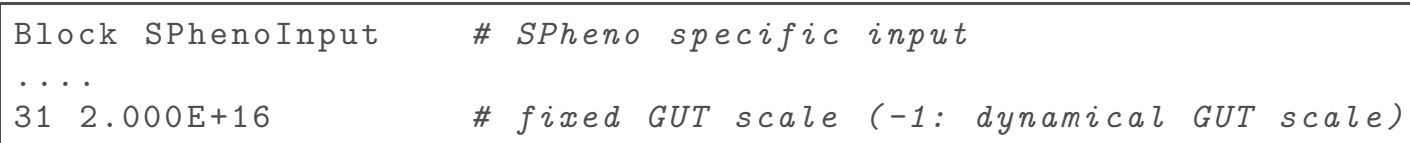

while with

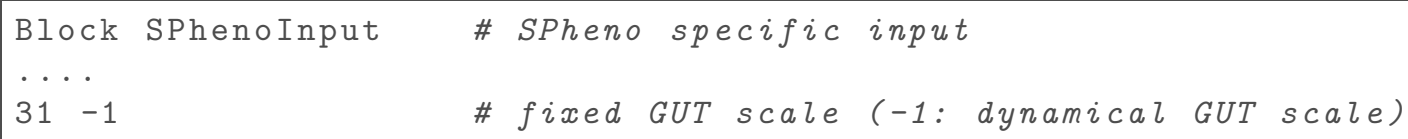

the condition defined by ConditionGUTscale is used to get a dynamical GUT scale.

\subsection{Interfacing SPheno and Monte Carlo Tools}

The information obtained by SPheno can be directly used together with the corresponding SARAH model files for CalcHep, MadGraph or WHIZARD to make Monte Carlo (MC) studies. We show in the following how the information can be exchanged between SPheno and the different tools.

\subsubsection{CalcHep}

In the case of CalcHep it is necessary to make sure that SARAH writes the corresponding model files using the SLHA+ functionality of CalcHep [59] which enables CalcHep to read SLHA files:

MakeCHep [SLHAinput $\rightarrow$ True];

This is also the default option of MakeCHep [] and it is used if the user hasn't changed the default settings before. In this case, CalcHep extracts from the SPheno spectrum file the information about all parameters, masses and rotation matrices. For this purpose the spectrum file must be located in the directory in which $\mathrm{n} \_c a l c h e p$ is executed. Of course, if the SARAH model files for CalcHep are used together with MicrOmegas, it is also sufficient to copy the SPheno spectrum file to the directory of the MicrOmegas main file which is used for the dark matter study.

\subsubsection{MadGraph}

To use the information obtained by SPheno together with MadGraph, replace the file param_card.dat in [\$MadGraph-Directory]/[\$Project]/Cards/ by the SPheno spectrum file. Here, [\$Project] is the MadGraph subdirectory which has been generated for the current MC study.

MadGraph has problems with reading the HiggsBounds specific blocks in the SPheno spectrum file (HiggsBoundsInputHiggsCouplingsFermions and HiggsBoundsInputHiggsCouplingsBosons). Therefore, either the output of this information must be disabled by setting

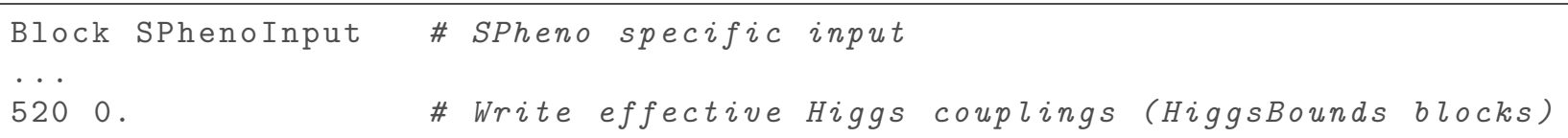


in the Les Houches input file of SPheno, or the blocks must be deleted before running MadGraph.

There is another subtlety: SARAH initializes by default all unknown parameters with 0 in the UFO file. Therefore, it can happen that MadGraph complains about a division by 0 when it generates the output and calculates internal parameters before loading a spectrum file. If this happens the user should put a non zero initialization value in parameters.py for the corresponding parameter.

\subsubsection{WHIZARD}

To link WHIZARD and SPheno, all SPheno modules created by SARAH write the information about the parameter and masses into an additional file called WHIZARD.par. [\$Model] in the WHIZARD specific format. To get this output, the following flag has to be set in the Les Houches input file of SPheno

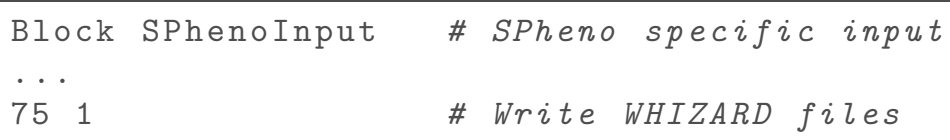

The parameter file can then be included in the Sindarin input file for WHIZARD via

include("[\$SPheno-Directory]/WHIZARD.par. [\$MODEL]")

\section{Vevacious}

Vevacious [38] is a new tool to check for the global minimum of the one-loop effective potential for a given model allowing for a particular set of non-zero VEVs. The importance of these checks has recently be demonstrated at the example of color and charge breaking minima in the MSSM [60]. As input Vevacious needs the tadpole equations, the polynomial part of the potential and all mass matrices for the model assuming that all allowed VEVs are non-zero. This means that, for instance, if stau VEVs should be studied a common basis of all uncolored scalars and fermions has to be defined and also the neutral gauge boson will mix with the $W$ bosons. Usually, these modifications of the SARAH model file are easily done and examples for the MSSM with different charge and color breaking scenarios are included in [\$SAHRAH-Directory]/Models/CCB-MSSM. For a detailed discussion of the input format of Vevacious we refer to Ref. [38].

The model files for Vevacious for a model are generated after loading SARAH and initializing the model by the command MakeVevacious []:

$<<$ \$SARAH-Directory ] SARAH . m;

Start [" [\$MODEL]"];

MakeVevacious [Options];

The following options are possible

- ComplexParameters, Value: list of parameters, Default: \{\} :

By default, all parameters are assumed to be real when writing the Vevacious input files. However, the user can define those parameters which should be treated as complex.

- IgnoreParameters, Value: list of parameters, Default: \{\} :

The user can define a list of parameters which should be set to zero when writing the Vevacious input.

- OutputFile, Value: String, Default: [\$Model].vin:

The name used for the output file.

- Scheme, Value: DR or MS, Default: DR for SUSY models, MS for non-SUSY models:

Defines, if as renormalization scheme $\overline{\mathrm{DR}}^{\prime}$ or $\overline{\mathrm{MS}}$ should be used. See for details of the effective potential in the different schemes Ref. 61]. 
The first two options allow to treat parameters differently in the Vevacious output as defined in the SARAH model file. The reason is that the evaluation of a parameter point with Vevacious can be very time consuming and subdominant terms can be neglected using these options. That might speed up the run Vevacious, what is however not yet quantified.

\section{Conclusion}

We have presented the new features in SARAH 4. The focus has been on a largely improved and extended support of non-SUSY models. In particular, the complete two-loop RGEs for a general quantum field theory have been implemented. We have shown how SPheno can be used to calculate the mass spectrum and other observables for non-SUSY models and how this information is passed to MC tools. By linking Susyno, the handling of Lie groups has been generalized and models with color sextets can be treated in a way suitable for MC tools. Also the definition of global symmetries is now possible. Finally, the interface to Vevacious allows to perform checks for the global minimum in a huge variety of SUSY and non-SUSY models.

\section{Acknowledgments}

We are in debt to Renato Fonseca for providing us to the powerful routines of Susyno for the handling of Lie groups. We thank Daniel Busbridge, Eliel Camargo, Philip Diessner, Mark Goodsell, Wojciech Kotlarski, Manuel Krauss, Florian Lyonnet, Moritz McGarrie, Kilian Nickel, Ben O'Leary, Werner Porod, Ingo Schienbein, Kai Schmidt-Hoberg, Ken Van Tilburg, Alexander Voigt, and Akin Wingerter for their feedback, fruitful discussions and helpful suggestions.

\section{Appendix A. Other Improvements in SARAH 4}

\section{Appendix A.1. Gauge-Gravity Anomalies}

Since version 2 SARAH checks for all triangle gauge anomalies as well as Witten anomalies for the given particle content in the considered model. These checks have now be extended and also gauge-gravity anomalies are included.

\section{Appendix A.2. Definition of complex and aligned Vacuum Expectation Values}

The general definition of the decomposition of a complex scalar field into its real components and a VEV reads in SARAH

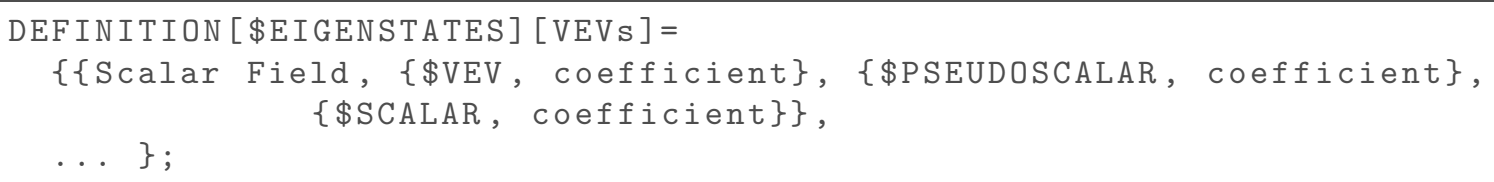

Here, \$VEV, \$PSEUDOSCALAR and \$SCALAR are the names chosen for the different components. Hence, for the two neutral Higgs doublets after electroweak symmetry breaking (EWSB) this reads

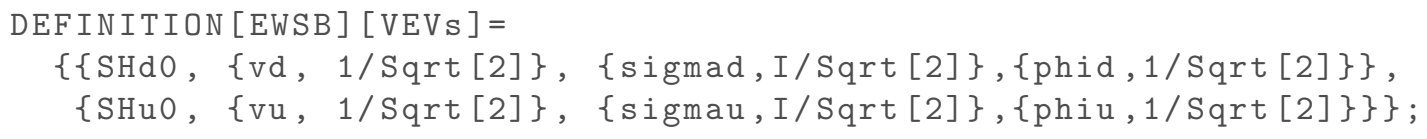

Two new possibilities have been added to the definition of VEVs in SARAH 4: the VEVs can be aligned, i.e. it is possible that only specific generations of a scalar field receive a VEV and the real and imaginary parts of the VEVs can be defined separately. Both changes are especially useful for the Vevacious output presented in 6 . 


\section{Appendix A.2.1. Aligned VEVs}

The standard definition of a model with broken electric charge due to charged slepton VEVs looks like

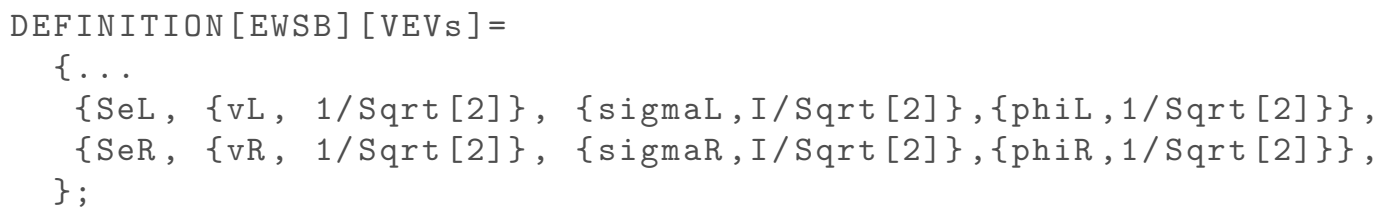

With this definition, all three generations of left and right sleptons would get a VEV. However, usually one is only interested in the case that staus receive VEVs. To restrict the possibility to obtain a VEV to the third generation the following syntax is used

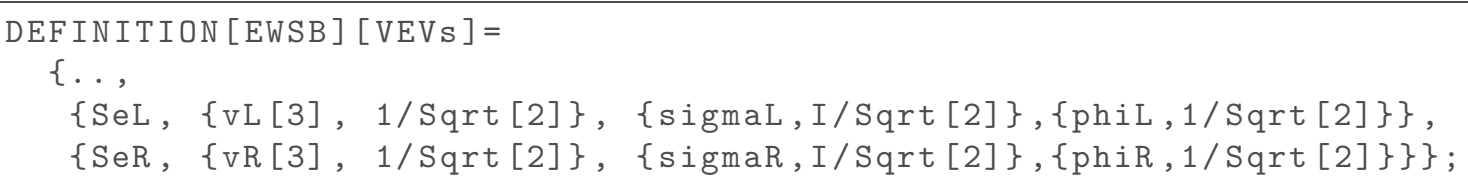

If one wants to consider smuon and stau VEVs, vL $[2,3]$, vR $[2,3]$ can be used.

\section{Appendix A.2.2. Complex VEVs}

To define complex VEVs, it has been possible in previous versions of SARAH to give the phase as last argument:

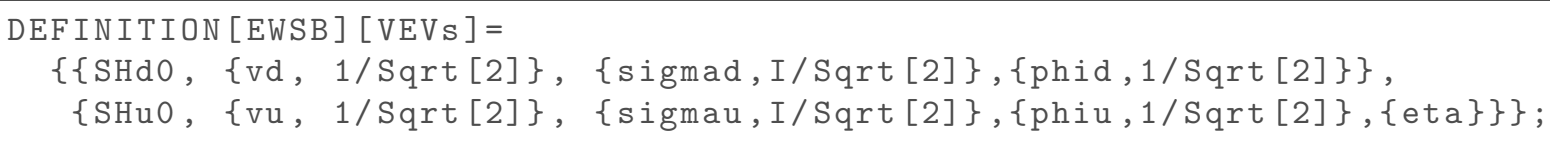

This is understood as $H_{u}^{0} \rightarrow \frac{\exp (i \eta)}{\sqrt{2}}\left(v_{u}+i \sigma_{u}+\phi_{u}\right)$. In SARAH 4, another possibility to define complex VEVs is to use

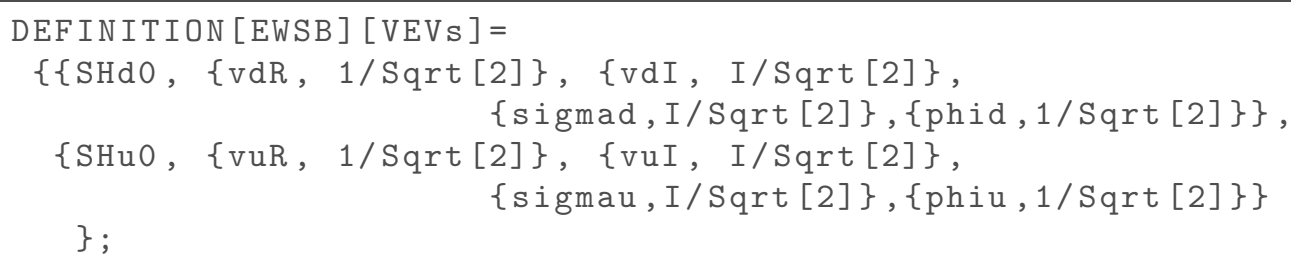

which is understood as

$$
H_{d}^{0} \rightarrow \frac{1}{\sqrt{2}}\left(v_{d}^{R}+i v_{d}^{I}+i \sigma_{d}+\phi_{d}\right), \quad H_{u}^{0} \rightarrow \frac{1}{\sqrt{2}}\left(v_{u}^{R}+i v_{u}^{I}+i \sigma_{u}+\phi_{u}\right) .
$$

This format has the advantage that the tree-level tadpole equations are also in the complex case polynomials in the VEVs. Therefore, they can be solved numerically with dedicated codes like HOM4PS2 [62] which is used by Vevacious.

\section{Appendix A.3. Numerical Solutions of Tadpole Equations in SPheno}

There has been so far a major restriction in the definition of the properties of the SPheno code: the tadpole equations have been solved on the SARAH side using the Solve command of Mathematica. Because of this it was necessary to choose a set of parameters for which an analytical solution of the tadpole equations exists. In the new version, this constrain is no longer present, but the tadpole equations can be solved numerically during the run of SPheno. The numerical solution is based on a Broydn method and finds the 
solution which is the closest one to a given starting point. This option needs at least SPheno 3.2.4.

We demonstrate the possibility to create SPheno modules using a numerical solution of the tadpole equations at the example of the MSSM. To use a numerical solution and to define the starting point, SPheno.m has to contain the following entries:

ParametersToSolveTadpoles $=\{\backslash[\mathrm{Mu}], \mathrm{B}[\backslash[\mathrm{Mu}]]\}$;

NumericalsolutionTadpoleEquations = True;

InitializationTadpoleParameters $=\left\{\backslash[\mathrm{Mu}] \rightarrow \mathrm{mo}, \mathrm{B}[\backslash[\mathrm{Mu}]]->\mathrm{m} 0^{\wedge} 2\right\}$;

The first line is the same as for the analytical approach and defines that the tadpole equations have to be solved with respect to $\mu$ and $B_{\mu}$. Without the other two lines, Mathematica's function Solve would be used for this and the analytical solution would be exported to the Fortran code. However, due to the second line the attempts to solve the tadpole equations inside Mathematica are skipped. The third line assumes that $\mu$ is $O\left(m_{0}\right)$ and $B_{\mu}$ is $O\left(m_{0}^{2}\right)\left(m_{0}\right.$ is the unified scalar mass at the GUT scale) and the numerical routines use those values as initialization. Of course, other possible and reasonable choices would have been to relate $\mu, B_{\mu}$ with the running soft-breaking terms of the Higgs

InitializationTadpoleParameters $=\{\backslash[\mathrm{Mu}]->\operatorname{Sqrt}[\mathrm{mHd} 2], \mathrm{B}[\backslash[\mathrm{Mu}]]->\mathrm{mHd} 2\}$;

or to use fixed values

InitializationTadpoleParameters $=\left\{\backslash[\mathrm{Mu}]->10^{\wedge} 3, \mathrm{~B}\left[\backslash[\mathrm{Mu}]->10^{\wedge} 6\right\}\right.$;

Usually, the time needed to find the solution changes only slightly with the chosen initialization values as long as they are not completely off.

Note, all choices above would only find the solution with positive $\mu$. Since an analytical solution, if it exists, contains all possible extrema, this is usually the preferred method. Moreover, one has to be careful because the numerical approach only finds one minimum which must not be the global one. This could especially happen if one solves with respect to some VEVs which enter the equations with third power. To check if the found minimum is really the global one or to calculate the life time of a metastable point one can use Vevacious.

\section{Appendix A.4. Running VEVs including Gauge Dependence}

SARAH 4 does not only calculate the one- and two-loop $\beta$-functions for all VEVs present in the model, but it includes also the gauge dependence of these RGEs. This is done by including the results of Ref. [55]. To express the gauge dependence a variable $\mathrm{X} i$ is introduced and $\mathrm{X} i$ equal to 0 corresponds to Landau and equal to 1 to Feynman gauge. With this convention the one- and two-loop $\beta$-function for $v_{d}$ in the MSSM reads now (BetaVEV $[[1]])$

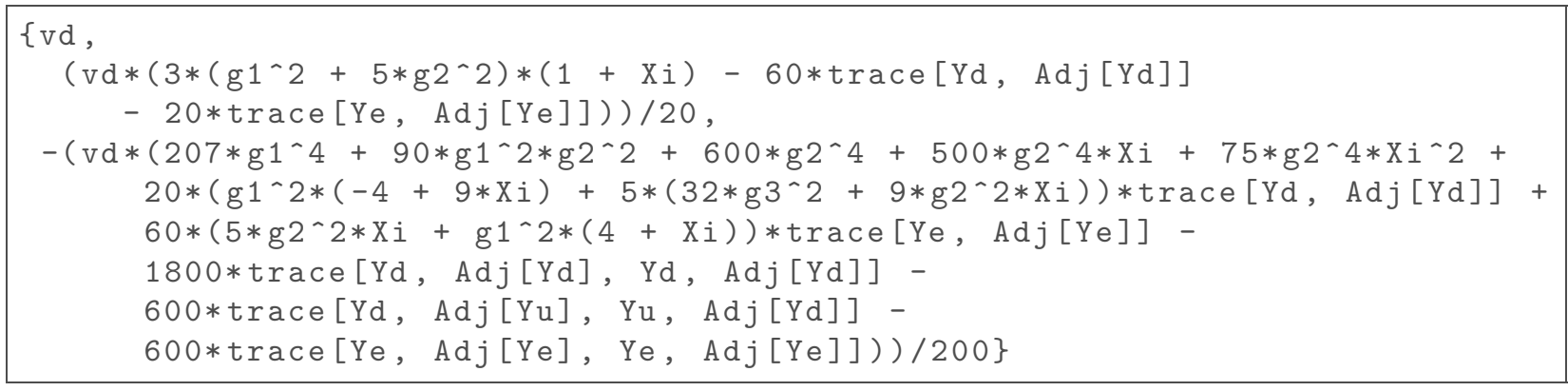




\section{Appendix A.5. Loop Corrections}

SARAH calculates the one-loop corrections to the 1- and 2-point functions which can be used for a calculation of the one-loop corrected mass spectrum. This is done in 't Hooft gauge and so far only the $\overline{\mathrm{DR}}$ scheme has been used. However, it is more convenient to perform these calculations for non-SUSY models in the $\overline{\mathrm{MS}}$ scheme. The results between both scheme differ by a constant term which is now reflected in the variable rMS introduced in SARAH 4. $r M S=0$ gives the results in $\overline{\mathrm{DR}}$ scheme and rMS $=1$ corresponds to $\overline{\mathrm{MS}}$ scheme.

\section{Appendix B. Summary of Commands}

We only list here the most important commands with their main options available in SARAH. For more details we refer to the manual.

\section{Appendix B.1. Tree Level Relations}

- Mass matrices

The mass matrix for a particle (Particle) is returned by

$$
\text { MassMatrix [Particle] }
$$

- Tadpole equations

The tadpole equation corresponding to a scalar (Scalar) is printed by using

$$
\text { TadpoleEquation [Scalar] }
$$

- Vertices

To calculate the vertices for a list of external states (Particles) use

$$
\text { Vertex [\{Particles\}, Options]; }
$$

The options define the considered eigenstates (Eigenstates $\rightarrow$ EWSB/GaugeES) as well as the treatment of dependences among parameters (UseDependences $\rightarrow$ False/True).

All vertices for a set of eigenstates are calculated at once by

MakeVertexList [\$EIGENSTATES, Options];

Here, first the eigenstates (\$EIGENSTATES: GaugeES, EWSB) are defined and as option it can be defined if only specific generic classes should be considered (e.g. GenericClasses -> FFS).

\section{Appendix B.2. Loop Calculations}

- Renormalization Group Equations

The calculation of the RGEs at the one- and two-loop level can be started after the initialization of a model via

\section{CalcRGEs [Options];}

See sec. 4 for the different options and the output for non-SUSY models. The output for SUSY models is saved in the following arrays:

- Gij: Anomalous dimensions of all chiral superfields

- BetaWijkl: Quartic superpotential parameters

- BetaYijk: Trilinear superpotential parameters

- BetaMuij: Bilinear superpotential parameters

- BetaLi: Linear superpotential parameters 
- BetaQijkl: Quartic soft-breaking parameters

- BetaTijk: Trilinear soft-breaking parameters

- BetaBij: Bilinear soft-breaking parameters

- BetaSLi: Linear soft-breaking parameters

- Betam2ij: Scalar squared masses

- BetaMi: Majorana Gaugino masses

- BetaGauge: Gauge couplings

- BetaVEVs: VEVs

- BetaDGi: Dirac gaugino mass terms

- One-loop Tadpoles and Self-Energies Loop corrections are calculated via

CalcLoopCorrections [\$EIGENSTATES];

As argument only the considered eigenstates (e.g. \$EIGENSTATES=EWSB) have to be defined. The results are saved in the variables Tadpoles1LoopSums [\$EIGENSTATES] and SelfEnergy1LoopSum [\$EIGENSTATES] as sums of all contributions, or as list of the different contributions in Tadpoles1LoopList [\$EIGENSTATES] and SelfEnergy1LoopList [\$EIGENSTATES].

Appendix B.3. Model Files and $\mathrm{E}^{A} T_{E} X$ Output

- UFO format

Model files in the UFO format are written by

MakeUFO [Options]

As option it can be defined, if specific generic classes (like four scalar interactions) should be excluded in the output (e.g. Exclude $\rightarrow\{$ SSSS $\}$ ).

- CalcHep/CompHep

Model files for CalcHep which also can be used with MicrOmegas are generated by

MakeCHep [Options]

The options define for instance if the CompHep format should be used instead of the CalcHep format (CompHep->False/True), if Feynman gauge and/or CP violation should be included (FeynmanGauge $\rightarrow$ True/False, CPViolation -> True/False) and how the masses of fields should be obtained by CalcHep (SLHAinput, CalculateMasses, RunSPhenoViaCalcHep).

- WHIZARD

The model files for WHIZARD and O'Mega are generated by

MakeWHIZARD [Options]

The options can be used to define the generic classes of vertices which should be excluded (e.g. Exclude $\rightarrow\{$ SSSS $\}$ ), and the maximal number of couplings per file (MaximalCouplingsPerFile $\rightarrow \#$ ).

- FeynArts/FormCalc Model files for FeynArts are written by

MakeFeynArts [Options]

As option the treatment of counter terms can be defined (AddCounterTerms $\rightarrow$ True/False)

- Vevacious The model files for Vevacious are written by 
MakeVevacious [Options]

For the different options, see sec. 6 .

- SPheno

To output the SPheno code, the command

MakeSPheno[Options];

has to be used. The options define the file which contains the necessary input for a custom made SPheno module (InputFile $\rightarrow$ \$FILENAME), defines the standard compiler (StandardCompiler $\rightarrow>$ \$COMPILER), and if the results from previous calculations should be used (ReadLists $\rightarrow>$ True/False)

- HATEX:

All derived information (mass matrices, vertices, RGEs, loop corrections) can be exported to $\mathrm{LT}_{\mathrm{E}} \mathrm{X}$ to get the expressions in a human readable form. This is done via

MakeTeX [Options]

The options define if Feynman diagrams should be painted with FeynMF 63] for all vertices

(FeynmanDiagrams $\rightarrow$ True/False), and if SARAH specific information (e.g. internal names for fields and parameters) should be written as well (WriteSARAH $\rightarrow$ True/False).

\section{Appendix C. Models}

\section{Appendix C.1. Supersymmetric Models}

- Minimal supersymmetric standard model

- With general flavor and CP structure (MSSM)

- Without flavor violation (MSSM/NoFV)

- With explicit CP violation in the Higgs sector (MSSM/CPV)

- In SCKM basis (MSSM/CKM)

- Singlet extensions:

- Next-to-minimal supersymmetric standard model (NMSSM, NMSSM/NoFV, NMSSM/CPV, NMSSM/CKM)

- near-to-minimal supersymmetric standard model (near-MSSM)

- General singlet extended, supersymmetric standard model (SMSSM)

- Triplet extensions

- Triplet extended MSSM (TMSSM)

- Triplet extended NMSSM (TNMSSM)

- Models with $R$-parity violation

- bilinear RpV (MSSM-RpV/Bi)

- Lepton number violation (MSSM-RpV/LnV)

- Only trilinear lepton number violation (MSSM-RpV/TriLnV)

- Baryon number violation (MSSM-RpV/BnV)

$-\mu \nu \operatorname{SSM}$ (munuSSM)

- Additional $U(1)^{\prime} s$ 
- U(1)-extended MSSM (UMSSM)

- secluded MSSM (secluded-MSSM)

- minimal $B-L$ model (B-L-SSM)

- minimal singlet-extended $B-L$ model (N-B-L-SSM)

- SUSY-scale seesaw extensions

- inverse seesaw (inverse-Seesaw)

- linear seesaw (LinSeesaw)

- singlet extended inverse seesaw (inverse-Seesaw-NMSSM)

- inverse seesaw with $B-L$ gauge group (B-L-SSM-IS)

- minimal $U(1)_{R} \times U(1)_{B-L}$ model with inverse seesaw (BLRinvSeesaw)

- Models with Dirac Gauginos

- MSSM/NMSSM with Dirac Gauginos (DiracGauginos)

- minimal $R$-Symmetric SSM (MRSSM)

- High-scale extensions

- Seesaw 1 - 3 (SU(5) version), (Seesaw1,Seesaw2,Seesaw3)

- Left/right model ( $\Omega \mathrm{LR}$ ) (Omega)

Appendix C.2. Non-Supersymmetric Models

- Standard Model (SM) (SM), Standard model in CKM basis (SM/CKM)

- inert Higgs doublet model (Inert)

- B-L extended SM (B-L-SM)

- B-L extended SM with inverse seesaw (B-L-SM-IS)

- SM extended by a scalar color octet (SM-8C)

- Two Higgs doublet model (THDM)

- Singlet extended SM (SSM)

Appendix D. The Model File of the NMSSM in SARAH 4

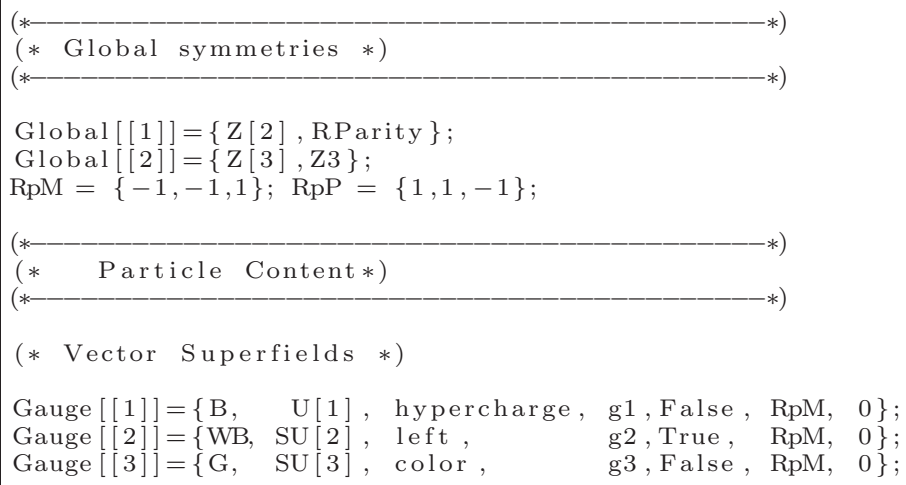




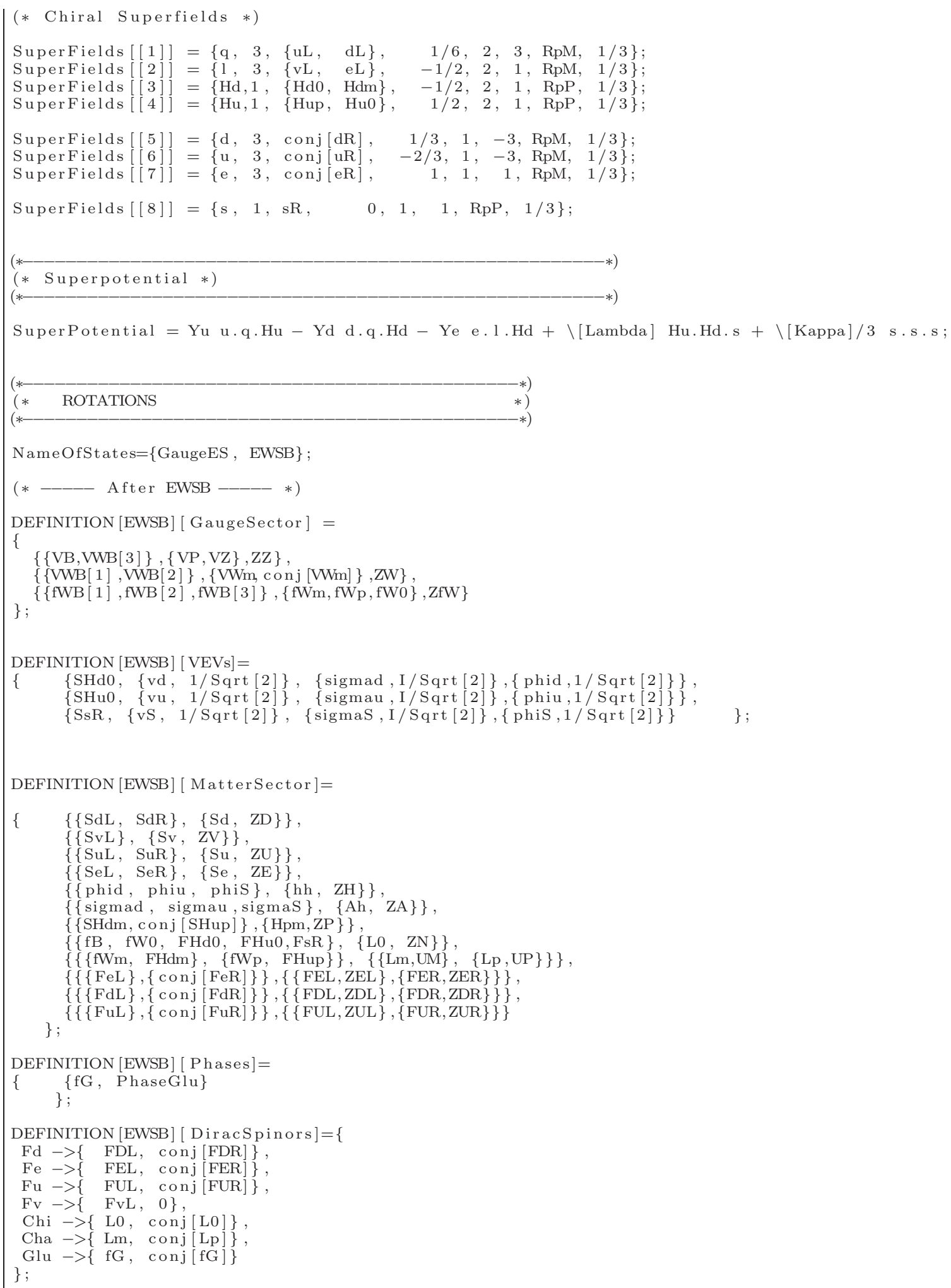


Appendix E. The Model File of the SM in SARAH 4

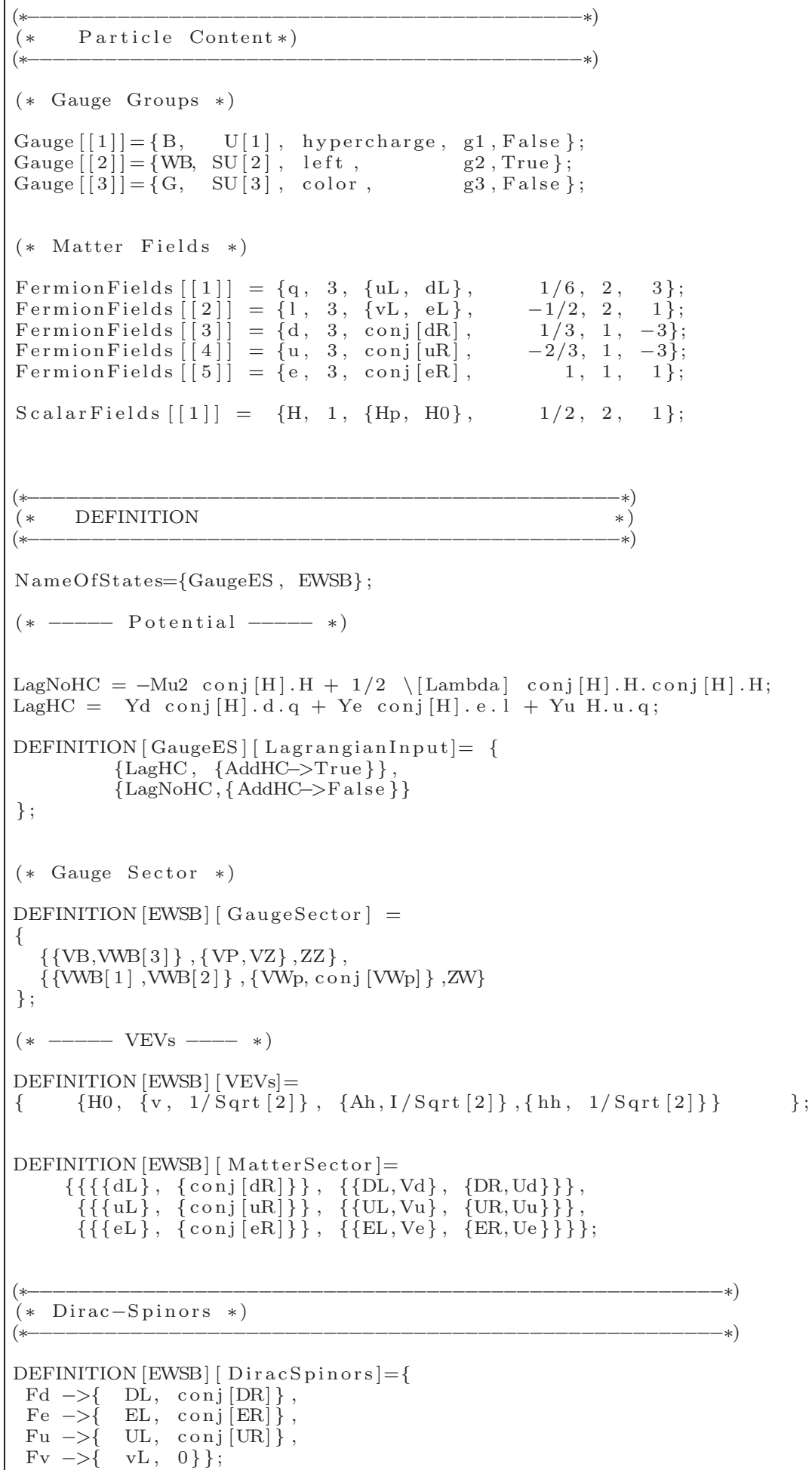




\section{References}

[1] F. Staub, "SARAH," 2008.

[2] F. Staub, "From Superpotential to Model Files for FeynArts and CalcHep/CompHep," Comput.Phys.Commun., vol. 181, pp. 1077-1086, 2010.

[3] F. Staub, "Automatic Calculation of supersymmetric Renormalization Group Equations and Self Energies," Comput.Phys.Commun., vol. 182, pp. 808-833, 2011.

[4] F. Staub, "SARAH 3.2: Dirac Gauginos, UFO output, and more," Computer Physics Communications, vol. 184, pp. pp. 1792-1809, 2013

[5] K. Ender, T. Graf, M. Muhlleitner, and H. Rzehak, "Analysis of the NMSSM Higgs Boson Masses at One-Loop Level," Phys.Rev., vol. D85, p. 075024, 2012.

[6] T. Graf, R. Grober, M. Muhlleitner, H. Rzehak, and K. Walz, "Higgs Boson Masses in the Complex NMSSM at One-Loop Level," JHEP, vol. 1210, p. 122, 2012.

[7] A. Kaminska, G. G. Ross, and K. Schmidt-Hoberg, "Non-universal gaugino masses and fine tuning implications for SUSY searches in the MSSM and the GNMSSM," 2013.

[8] J. List and B. Vormwald, "Bilinear R Parity Violation at the ILC - Neutrino Physics at Colliders," 2013.

[9] H. Dreiner, K. Nickel, and F. Staub, " $B_{s, d}^{0} \rightarrow \mu \bar{\mu}$ and $B \rightarrow X_{s} \gamma$ in the R-parity violating MSSM," 2013.

[10] A. Abada, A. Figueiredo, J. Romao, and A. Teixeira, "Probing the supersymmetric type III seesaw: LFV at low-energies and at the LHC," JHEP, vol. 1108, p. 099, 2011.

[11] P. Bhupal Dev, S. Mondal, B. Mukhopadhyaya, and S. Roy, "Phenomenology of Light Sneutrino Dark Matter in cMSSM/mSUGRA with Inverse Seesaw," JHEP, vol. 1209, p. 110, 2012.

[12] V. De Romeri and M. Hirsch, "Sneutrino Dark Matter in Low-scale Seesaw Scenarios," JHEP, vol. 1212, p. 106, 2012.

[13] J. Esteves, J. Romao, M. Hirsch, A. Vicente, W. Porod, et al., "LHC and lepton flavour violation phenomenology of a left-right extension of the MSSM," JHEP, vol. 1012, p. 077, 2010.

[14] M. E. Krauss, W. Porod, and F. Staub, "SO(10) inspired gauge-mediated supersymmetry breaking," Phys.Rev., vol. D88, p. $015014,2013$.

[15] B. O'Leary, W. Porod, and F. Staub, "Mass spectrum of the minimal SUSY B-L model," JHEP, vol. 1205 , p. 042, 2012.

[16] M. Hirsch, W. Porod, L. Reichert, and F. Staub, "Phenomenology of the minimal supersymmetric $U(1)_{B-L} \times U(1)_{R}$ extension of the standard model," Phys.Rev., vol. D86, p. 093018, 2012.

[17] C. Frugiuele, T. Gregoire, P. Kumar, and E. Ponton, "'L=R' - $U(1)_{R}$ as the Origin of Leptonic 'RPV'," JHEP, vol. 1303, p. $156,2013$.

[18] K. Benakli, M. D. Goodsell, and F. Staub, "Dirac Gauginos and the 125 GeV Higgs," JHEP, vol. 1306, p. 073, 2013.

[19] D. S. Alves, P. J. Fox, and N. Weiner, "Supersymmetry with a Sister Higgs," 2012.

[20] A. Pukhov, "CalcHEP 2.3: MSSM, structure functions, event generation, batchs, and generation of matrix elements for other packages," 2004.

[21] E. Boos, M. Dubinin, V. Ilyin, A. Pukhov, and V. Savrin, "CompHEP: Specialized package for automatic calculations of elementary particle decays and collisions," 1994.

[22] T. Hahn, "Generating Feynman diagrams and amplitudes with FeynArts 3," Comput.Phys.Commun., vol. 140, pp. 418$431,2001$.

[23] T. Hahn, "FormCalc 6," PoS, vol. ACAT08, p. 121, 2008.

[24] W. Kilian, T. Ohl, and J. Reuter, "WHIZARD: Simulating Multi-Particle Processes at LHC and ILC," Eur.Phys.J., vol. C71, p. 1742, 2011.

[25] M. Moretti, T. Ohl, and J. Reuter, "O'Mega: An Optimizing matrix element generator," 2001.

[26] C. Degrande, C. Duhr, B. Fuks, D. Grellscheid, O. Mattelaer, et al., "UFO - The Universal FeynRules Output," Comput.Phys.Commun., vol. 183, pp. 1201-1214, 2012.

[27] J. Alwall, M. Herquet, F. Maltoni, O. Mattelaer, and T. Stelzer, "MadGraph 5 : Going Beyond," JHEP, vol. 1106, p. 128, 2011.

[28] G. Cullen, N. Greiner, G. Heinrich, G. Luisoni, P. Mastrolia, et al., "Automated One-Loop Calculations with GoSam," Eur.Phys.J., vol. C72, p. 1889, 2012.

[29] S. Gieseke, A. Ribon, M. H. Seymour, P. Stephens, and B. Webber, "Herwig++ 1.0: An Event generator for e+ eannihilation," JHEP, vol. 0402, p. 005, 2004.

[30] S. Gieseke, D. Grellscheid, K. Hamilton, A. Ribon, P. Richardson, et al., "Herwig++ 2.0 Release Note," 2006.

[31] J. Bellm, S. Gieseke, D. Grellscheid, A. Papaefstathiou, S. Platzer, et al., "Herwig++ 2.7 Release Note," 2013.

[32] W. Porod, "SPheno, a program for calculating supersymmetric spectra, SUSY particle decays and SUSY particle production at e+ e- colliders," Comput.Phys.Commun., vol. 153, pp. 275-315, 2003.

[33] W. Porod and F. Staub, "SPheno 3.1: Extensions including flavour, CP-phases and models beyond the MSSM," Comput.Phys.Commun., vol. 183, pp. 2458-2469, 2012.

[34] P. Bechtle, O. Brein, S. Heinemeyer, G. Weiglein, and K. E. Williams, "HiggsBounds: Confronting Arbitrary Higgs Sectors with Exclusion Bounds from LEP and the Tevatron," Comput.Phys.Commun., vol. 181, pp. 138-167, 2010.

[35] P. Bechtle, O. Brein, S. Heinemeyer, G. Weiglein, and K. E. Williams, "HiggsBounds 2.0.0: Confronting Neutral and Charged Higgs Sector Predictions with Exclusion Bounds from LEP and the Tevatron," Comput.Phys.Commun., vol. 182, pp. 2605-2631, 2011.

[36] P. Bechtle, S. Heinemeyer, O. Stl, T. Stefaniak, and G. Weiglein, "HiggsSignals: Confronting arbitrary Higgs sectors with measurements at the Tevatron and the LHC," 2013. 
[37] R. M. Fonseca, "Calculating the renormalisation group equations of a SUSY model with Susyno," Comput.Phys.Commun., vol. 183, pp. 2298-2306, 2012.

[38] J. Camargo-Molina, B. O'Leary, W. Porod, and F. Staub, "Vevacious: A Tool For Finding The Global Minima Of One-Loop Effective Potentials With Many Scalars," 2013.

[39] C. L. Wainwright, "CosmoTransitions: Computing Cosmological Phase Transition Temperatures and Bubble Profiles with Multiple Fields," Comput.Phys.Commun., vol. 183, pp. 2006-2013, 2012.

[40] A. Semenov, "LanHEP: A package for automatic generation of Feynman rules from the Lagrangian," Comput.Phys.Commun., vol. 115, pp. 124-139, 1998.

[41] A. Semenov, "LanHEP: A Package for automatic generation of Feynman rules in field theory. Version 2.0," 2002.

[42] A. Semenov, "LanHEP: A Package for the automatic generation of Feynman rules in field theory. Version 3.0," Comput.Phys.Commun., vol. 180, pp. 431-454, 2009.

[43] A. Semenov, "LanHEP - a package for automatic generation of Feynman rules from the Lagrangian. Updated version 3.1," 2010.

[44] N. D. Christensen and C. Duhr, "FeynRules - Feynman rules made easy," Comput.Phys.Commun., vol. 180, pp. 1614-1641, 2009.

[45] N. D. Christensen, C. Duhr, B. Fuks, J. Reuter, and C. Speckner, "Introducing an interface between WHIZARD and FeynRules," Eur.Phys.J., vol. C72, p. 1990, 2012.

[46] A. Bharucha, A. Goudelis, and M. McGarrie, "En-gauging Naturalness," 2013.

[47] S. P. Martin and M. T. Vaughn, "Two loop renormalization group equations for soft supersymmetry breaking couplings," Phys.Rev., vol. D50, p. 2282, 1994.

[48] R. M. Fonseca, M. Malinsky, W. Porod, and F. Staub, "Running soft parameters in SUSY models with multiple U(1) gauge factors," Nucl.Phys., vol. B854, pp. 28-53, 2012.

[49] M. D. Goodsell, "Two-loop RGEs with Dirac gaugino masses," JHEP, vol. 1301, p. 066, 2013.

[50] M. E. Machacek and M. T. Vaughn, "Two Loop Renormalization Group Equations in a General Quantum Field Theory. 1. Wave Function Renormalization," Nucl. Phys., vol. B222, p. 83, 1983.

[51] M. E. Machacek and M. T. Vaughn, "Two Loop Renormalization Group Equations in a General Quantum Field Theory. 2. Yukawa Couplings," Nucl. Phys., vol. B236, p. 221, 1984.

[52] M. E. Machacek and M. T. Vaughn, "Two Loop Renormalization Group Equations in a General Quantum Field Theory. 3. Scalar Quartic Couplings," Nucl. Phys., vol. B249, p. 70, 1985.

[53] M.-x. Luo, H.-w. Wang, and Y. Xiao, "Two-loop renormalization group equations in general gauge field theories," Phys. Rev., vol. D67, p. 065019, 2003.

[54] R. M. Fonseca, M. Malinsky, and F. Staub, "Renormalization group equations and matching in a general quantum field theory with kinetic mixing," 2013.

55] M. Sperling, D. Stckinger, and A. Voigt, "Renormalization of vacuum expectation values in spontaneously broken gauge theories," JHEP, vol. 1307, p. 132, 2013.

[56] F. Lyonnet, I. Schienbein, F. Staub, and A. Wingerter, "PyR@TE: Renormalization Group Equations for General Gauge Theories," 2013.

[57] H. Dreiner, K. Nickel, W. Porod, and F. Staub, "Full 1-loop calculation of $\operatorname{BR}\left(B_{s, d}^{0} \rightarrow \ell \bar{\ell}\right)$ in models beyond the MSSM with SARAH and SPheno," Comput.Phys.Commun., vol. 184, pp. 2604-2617, 2013.

[58] F. Staub, T. Ohl, W. Porod, and C. Speckner, "A Tool Box for Implementing Supersymmetric Models," Comput.Phys.Commun., vol. 183, pp. 2165-2206, 2012.

[59] G. Belanger, N. D. Christensen, A. Pukhov, and A. Semenov, "SLHAplus: a library for implementing extensions of the standard model," Comput.Phys.Commun., vol. 182, pp. 763-774, 2011.

[60] J. Camargo-Molina, B. O'Leary, W. Porod, and F. Staub, "Stability of the CMSSM against sfermion VEVs," 2013.

[61] S. P. Martin, "Two loop effective potential for a general renormalizable theory and softly broken supersymmetry," Phys.Rev., vol. D65, p. 116003, 2002.

[62] T. Lee, T. Li, and C. Tsai, "Hom4ps-2.0: a software package for solving polynomial systems by the polyhedral homotopy continuation method," Computing, vol. 83, no. 2, pp. 109-133, 2008.

[63] T. Ohl, "Feynmf: Drawing feynman diagrams with latex and metafont," 1997. 